\title{
Orbital pseudotumour as a presentation of paediatric ulcerative colitis
}

\author{
Justin Hung Tiong $\underline{T a n}^{1}$, MBBS, MRCPCH, Hui Ping $\underline{C h u^{2}}$, MBBS, MRCPCH, Lena Das ${ }^{1}$, MD, Thaschawee $\underline{\text { Arkachaisri }}^{1,3}$, MD, FACR
}

\begin{abstract}
A 2-year-old girl presented with a one-day history of acute-onset bilateral painful, swollen eyes and a two-month history of loose stools. Physical examination revealed a right eyelid swelling with proptosis. Magnetic resonance imaging revealed a right orbital pseudotumour. The patient responded well to treatment with intravenous antibiotics and nonsteroidal anti-inflammatory drugs. However, three weeks later, she was readmitted with a vasculitic lesion over her left upper chest, with mucous-bloody diarrhoea. Histopathology confirmed the diagnosis of ulcerative colitis. The patient was treated with intravenous pulse methylprednisolone and sulphasalazine. Two weeks after discharge, she was readmitted for cutaneous vasculitis and worsening diarrhoea. The patient's bowel and extraintestinal diseases resolved upon addition of infliximab to her treatment regimen. Her inflammatory markers also normalised. Azathioprine was subsequently added. Infliximab was discontinued after four doses and prednisolone was tapered off. The patient remained well without any flare-up after 24 months of follow-up.
\end{abstract}

Keywords: inflammatory bowel disease, orbital, paediatrics, pseudotumour, ulcerative colitis

\section{INTRODUCTION}

Inflammatory bowel disease (IBD) is an inflammatory condition that encompasses Crohn's disease and ulcerative colitis. Although it primarily affects the gastrointestinal tract, approximately $25 \%-35 \%$ of paediatric IBD patients develop extraintestinal manifestations, which commonly affect the mucocutaneous, articular and ocular systems. (1) Ocular diseases, mostly inflammatory in nature, occur in up to $4 \%$ of these patients, including those with asymptomatic uveitis. ${ }^{(2)}$ Apart from episcleritis and uveitis, orbital pseudotumour is rarely described in the literature. We herein report, to the best of our knowledge, the first case of orbital pseudotumour as a presentation of paediatric ulcerative colitis.

\section{CASE REPORT}

A 2-year-old girl was admitted to our hospital in November 2009 with a one-day history of acute-onset bilateral painful, swollen eyes, but with intact vision. She had loose stools for two months, which was initially thought to be due to lactose intolerance. There was no fever or associated sinusitis and her family history was unremarkable.

Physical examination revealed a pale, afebrile girl in no acute distress. Her weight and height were below the third percentile. Although both eyelids were red and swollen, the right eyelid was more severely swollen, with ptosis and proptosis (Fig. 1). There was no limited extraocular movement, facial tenderness, rhinorrhoea or nasal congestion, and the remainder of her examination was unremarkable.

Laboratory tests were significant for anaemia (haemoglobin $10.1 \mathrm{~g} / \mathrm{dL}$ ) and leucocytosis (white blood cell [WBC]17.40 × 10\% $/ \mathrm{L}$; neutrophils $72 \%$ ). The patient's platelet count was $479 \times 10^{9} / \mathrm{L}$ and

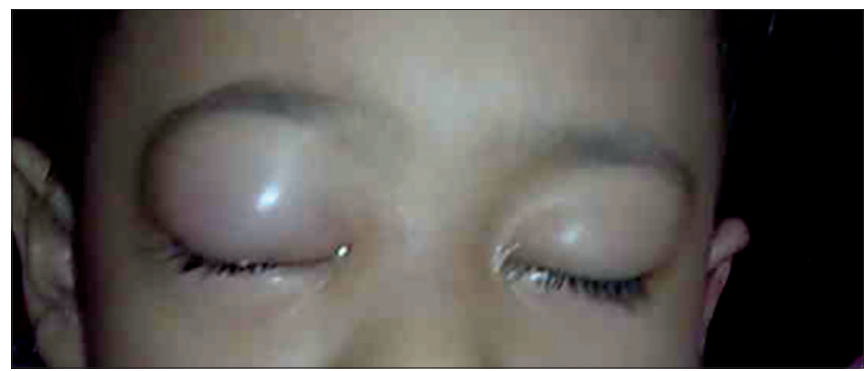

Fig. 1 Photograph shows bilateral eyelid swelling and erythema, which is more severe in the right eye.

erythrocyte sedimentation rate (ESR) was elevated at $60 \mathrm{~mm} / \mathrm{hr}$, while C-reactive protein (CRP) level was normal at $6.9 \mathrm{mg} / \mathrm{L}$. Thyroid function test was normal. Magnetic resonance (MR) imaging of the orbit showed the right eye proptosis with a stretched right optic nerve (Fig. 2). There was general swelling and an increased T2 signal of the right infraorbital region, down to the maxillary region, and more posteriorly toward the infratemporal fossa. The right extraocular muscles were swollen despite being stretched. There was minor postcontrast enhancement of the swollen fat and muscles, and no evidence of paranasal sinusitis or orbital bone erosion.

The patient's family refused a retro-orbital tissue biopsy. Thus, intravenous antibiotics and an anti-inflammatory dose of ibuprofen (40 mg/kg/day) were started. The patient's eye swelling and pain diminished after five days. In the ward, even before the initiation of ibuprofen, she had an episode of per rectal bleeding, which resolved spontaneously. She was then discharged with oral ibuprofen, which was continued until the subsequent admission three weeks later, when the patient was readmitted with a oneday history of a vasculitic lesion measuring $4 \mathrm{~cm} \times 4 \mathrm{~cm}$ over her left upper chest. The patient was found to have lost $3 \mathrm{~kg}$

${ }^{1}$ Rheumatology and Immunology Service, Department of Paediatric Subspecialties, KK Women's and Children's Hospital, ${ }^{2}$ Raffles Children's Centre, Raffles Hospital, ${ }^{3}$ Duke-NUS Graduate Medical School, Singapore

Correspondence: Dr Justin Hung Tiong Tan, Consultant, Rheumatology and Immunology Service, Department of Paediatric Subspecialties, KK Women's and Children's Hospital, 100 Bukit Timah Road, Singapore 229899. justin.tan.ht@kkh.com.sg 

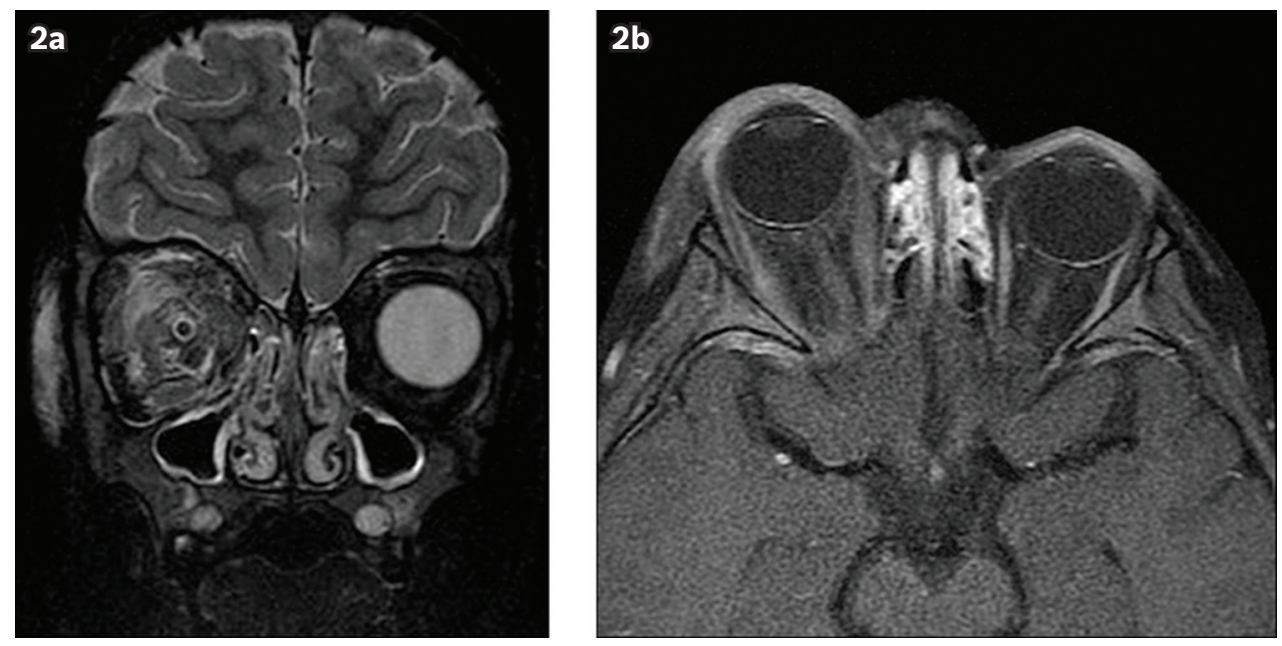

Fig. 2 MR images of the orbits show (a) the coronal STIR sequence revealing general swelling and increased signal of the right intraorbital fat, with extraocular muscle swelling despite being stretched; and (b) the axial, fat-suppressed T1-weighted sequence revealing minor contrast enhancement of the swollen fat and muscles of the right orbit.

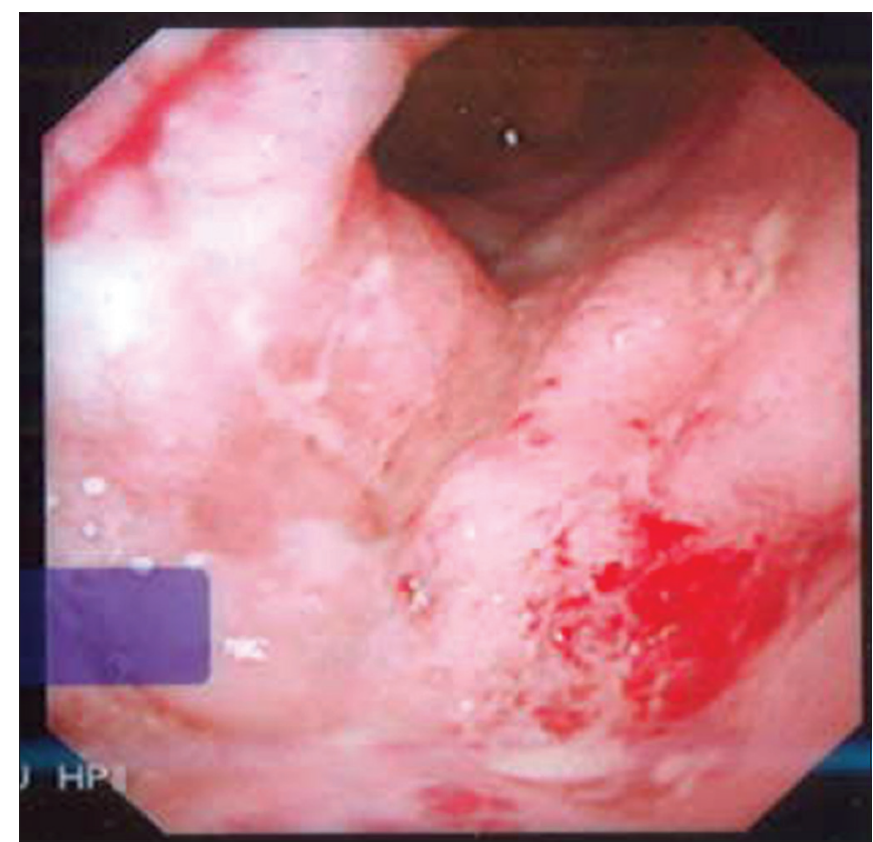

Fig. 3 Endoscopic image of the colon shows the oedematous mucosa with superficial ulcers, diffuse erythema, friability and loss of vascular pattern of the colon.

over two months. Mucous-bloody diarrhoea was observed. Laboratory tests showed worsening anaemia (haemoglobin $9.7 \mathrm{~g} / \mathrm{dL})$, elevated ESR $(25 \mathrm{~mm} / \mathrm{hr})$ and CRP level $(11.6 \mathrm{mg} / \mathrm{L})$, and a low serum albumin concentration (14 g/L). The patient's stool culture was negative. Abdominal ultrasonography showed marked thickening of the entire colon, sparing the small bowel. Oesophagogastroduodenoscopy and colonoscopy revealed diffuse erythema, granularity and loss of vascular pattern in the colon, and the absence of deep, fissuring ulcers (i.e. absence of cobblestone appearance) (Fig. 3).

Histopathology revealed chronic gastritis; mild, active duodenitis without ulcer; mild, active ileitis of the terminal ileum; and in the colonic mucosa, crypt architectural changes with branching, and crypt disarray. Cryptitis and crypt abscesses were found, but without granuloma. No ulcer or debris at the base of the ulcer was observed. There was no discontinuous inflammation, with intervening areas of normal bowel. No fissuring ulcer, stricture or fistula was seen, making Crohn's disease unlikely in this case, thus favouring the diagnosis of ulcerative colitis. Skin biopsy demonstrated leucocytoclastic vasculitis with acute septal/lobular panniculitis. The diagnosis of ulcerative colitis was thus made.

Intravenous pulse methylprednisolone $(30 \mathrm{mg} / \mathrm{kg} /$ dose for three days) and sulphasalazine (SSZ) were started. The patient was subsequently discharged with oral prednisolone $(1 \mathrm{mg} / \mathrm{kg} /$ day $)$ and SSZ. However, two weeks later, she was readmitted for worsening mucous-bloody diarrhoea and cutaneous vasculitis, and new onset bilateral ankle arthritis. Due to the patient's incomplete response to treatment for her ulcerative colitis and the presence of extraintestinal manifestations, infliximab was added to her treatment regimen. The patient responded well; bowel and extraintestinal diseases resolved, and inflammatory markers (i.e. ESR and CRP level) normalised. Azathioprine was added, while infliximab was discontinued after four doses, and prednisolone was tapered off. The patient was well without any flare-up after 24 months of follow-up.

\section{DISCUSSION}

Common ocular manifestations associated with IBD include conjunctivitis, episcleritis, blepharitis, scleritis, uveitis, cataracts, keratopathy, papillitis, vasculitis, optic neuritis, orbital myositis and orbital pseudotumour. ${ }^{(3,4)}$ Out of these, episcleritis, scleritis and uveitis are the most common ocular manifestations in adult IBD. ${ }^{(3,4)}$ However, little is known of IBD in children. Although ocular manifestations usually occur early in the disease course, it is uncommon to have ocular manifestation preceding IBD diagnosis.

Orbital pseudotumour is defined as a space-occupying inflammatory lesion within the orbit, mimicking a tumour. ${ }^{(5)}$ This accounts for $5 \%-16 \%$ of adult orbital diseases but its incidence in children is uncommon. ${ }^{(6)}$ In its idiopathic form, unilateral involvement is more common. Thus far, only 69 cases of 
childhood-onset orbital pseudotumour have been reported. ${ }^{(6)}$ Among these cases, swollen eyelids were present in $67 \%$, proptosis in $35 \%$, eyelid erythema in $17 \%$, and ocular movement restriction in $15 \%$. However, fever was not common, occurring in only $1.5 \%{ }^{(6)}$

Laboratory tests do not offer additional help for diagnosis; rather, they serve to rule out other diseases. ${ }^{(6,7)}$ Imaging studies, such as computed tomography and MR imaging, of the orbit typically show proptosis, contrast enhancement, infiltration of the orbital tissue, and extraocular muscle enlargement, with the absence of contiguous paranasal sinus disease and bony erosion or distortion of orbital contents. ${ }^{(7)}$ The definitive diagnosis of orbital pseudotumour is obtained by orbital biopsy. Patients who undergo orbital biopsy are, however, reported to suffer greater residua, which include residual visual acuity loss, higher risk of permanent and more severe exophthalmos. ${ }^{(5)}$ Currently, orbital biopsy is reserved for patients presenting with atypical clinical course or therapy resistance, and in patients where malignancies are strongly considered. In a patient presenting with painful and swollen eyelids with proptosis, differential diagnosis includes orbital infection, especially orbital cellulitis, which can be ruled out using normal sinus imaging. Other possible conditions include granulomatous diseases (e.g. Wegener's granulomatosis, sarcoidosis, Sjögren's syndrome and orbital tuberculosis), thyroid ophthalmopathy and malignancies (for which rhabdomyosarcoma remains the most common childhood orbital tumour). ${ }^{(5)}$ The latter is not associated with substantial inflammatory signs. ${ }^{(5)}$ Our patient's tuberculin skin test and T-spot test were both negative, and thyroid function test was normal. Anti-neutrophil cytoplasmic antibodies were also negative. There were no other manifestations in our patient that were compatible with other differential diagnoses.

Anti-inflammatory therapy remains the mainstay of orbital pseudotumour management. ${ }^{(5)}$ Corticosteroids are the drugs of choice. However, Mottow and Jakobiec ${ }^{(5)}$ reported that in their cohort of 29 children with orbital pseudotumour, seven non-prednisone-treated patients also showed considerable improvement in visual acuity. Interestingly, 11 patients were put on antibiotics, out of whom four exhibited relief of constitutional symptoms, and conjunctival injection was cleared in one. ${ }^{(5)}$ Our patient's orbital inflammation subsided with a combination of anti-inflammatory dose of nonsteroidal anti-inflammatory drugs and antibiotic therapy. We believe that our patient's orbital inflammatory disease was associated with active ulcerative colitis, as her mucous-bloody stools and weight loss preceded and remained ongoing during her ocular manifestations. She has been free of intestinal, ocular, cutaneous and articular diseases since discontinuation of infliximab and introduction of azathioprine on top of SSZ.

Orbital pseudotumour was previously reported in two teenage girls with Crohn's disease. ${ }^{(8,9)}$ Both patients had bilateral painful, proptotic eyes and a history of significant weight loss and bowel habit changes. One of them was treated with antibiotics, which resulted in resolution of her eye disease after two weeks. ${ }^{(9)}$ However, the disease recurred six months later, requiring corticosteroid therapy in order to sustain remission.

To the best of our knowledge, this is the first case report of orbital pseudotumour in a paediatric patient with ulcerative colitis. Orbital pseudotumour should be included as one of the differential diagnoses in ulcerative colitis patients with swollen eyelid and proptosis.

\section{REFERENCES}

1. Leichtner AM, Higuchi L. Epidemiology of ulcerative colitis. In: Goulet OJ, Kleinman R, Sherman P, et al, eds. Pediatric Gastrointestinal Disease. 4th ed. Ontario, Canada: BC Decker International, 2004.

2. Hofley P, Roarty J, McGinnity G, et al. Asymptomatic uveitis in children with chronic inflammatory bowel diseases. J Pediatr Gastroenterol Nutr 1993; 17:397-400.

3. Felekis T, Katsanos K, Kitsanou M, et al. Spectrum and frequency of ophthalmologic manifestations in patients with inflammatory bowel disease: a prospective single-center study. Inflamm Bowel Dis 2009; 15:29-34.

4. Yilmaz S, Aydemir E, Maden A, Unsal B. The prevalence of ocular involvement in patients with inflammatory bowel disease. Int J Colorectal Dis 2007; 22:1027-30.

5. Mottow LS, Jakobiec FA. Idiopathic inflammatory orbital pseudotumor in childhood. I. Clinical characteristics. Arch Ophthalmol 1978; 96:1410-7.

6. Kitei D, DiMario FJ Jr. Childhood orbital pseudotumor: case report and literature review. J Child Neurol 2008; 23:425-30.

7. Stevens JL, Rychwalski PJ, Baker RS, Kielar RS. Pseudotumor of the orbit in early childhood. J AAPOS 1998; 2:120-3.

8. Camfield PR, White M, Warner HA, Lythgoe C. Orbital pseudotumor and Crohn disease. J Pediatr 1982; 101:157-8.

9. Young RS, Hodes BL, Cruse RP, Koch KL, Garovoy MR. Orbital pseudotumor and Crohn disease. J Pediatr 1981; 99:250-2. 